\title{
Laminar Flame Speeds of Stratified Methane, Propane, and $n$-Heptane Flames
}

\author{
Xian Shi*, Jyh-Yuan Chen, Yulin Chen \\ Department of Mechanical Engineering, University of California - Berkeley, Berkeley, CA 94720, USA
}

\begin{abstract}
A numerical study on stratified flames of three hydrocarbon fuels, i.e., methane, propane and $n$-heptane, is conducted using a unsteady, compressible and reacting flow solver ASURF-Parallel. For each fuel, both fuel consumption speeds and flame front propagation speeds of a rich-to-lean stratified flame are compared to those of their corresponding homogeneous flames. For fuel consumption speeds, the methane/air stratified flame is overall faster than those of homogeneous flames due to chemical activities enhanced by key radicals and species from rich burnt gas mixtures. In contrast, stratified flames of both propane/air and $n$-heptane/air mixtures have lower fuel consumption speeds compared to their homogeneous flames on the rich side, due to reduced level of key radicals consumed by intermediate hydrocarbon species from rich burnt gases. For flame front propagation speeds, stratified flames of all three fuels are found faster than their corresponding homogeneous flames, due to consistently enhanced total heat release rate. Stronger enhancement of laminar flame speeds of stratified mixtures is observed in methane/air mixtures, compared to propane and $n$-heptane. Burnt gas of rich methane/air mixtures consists of relatively more molecular hydrogen, which assists fuel consumption and heat release at flame front. Moreover, molecular hydrogen has a stronger impact on laminar flame speeds of methane/air mixtures thus an even stronger enhancement on laminar flame speeds of methane/air stratified mixtures is observed. $\mathrm{H} / \mathrm{C}$ ratio along with local equivalence ratio at flame front are proposed to provide a unique identification of the exact mixture composition of stratified flames.
\end{abstract}

Keywords: Stratified flame, Laminar flame speed, Chemical effect, Preferential diffusion

$\begin{array}{ll}\text { Nomenclature } & \\ \phi_{f} & \text { Equivalence ratio at flame front } \\ \phi_{u} & \text { Equivalence ratio of unburnt mixture } \\ X_{k} & \text { Mole fraction of species } k \\ x_{f} & \text { Flame front location } \\ S_{c} & \text { Fuel consumption speed } \\ Y_{k} & \text { Mass fraction of species } k \\ \dot{\omega}_{F} & \text { Fuel consumption rate } \\ x & \text { Location } \\ t & \text { Time } \\ S_{L} & \text { Laminar flame speed relative to } \\ & \text { unburnt mixture } \\ S_{b} & \text { Flame front propagation speed } \\ \rho_{u} & \text { Density of unburnt mixture } \\ \rho_{b} & \text { Density of burnt mixture } \\ \delta_{S} & \text { Stratification thickness }\end{array}$

\section{Introduction}

Lean fuel-stratified combustion, as a promising modern engine combustion technology, yields high engine efficiency, low $\mathrm{NO}_{\mathrm{X}}$ emission and improvement in both ignitability and flame stability $[1,2,3,4,5]$. In addition, direct fuel injection which

\footnotetext{
${ }^{*}$ Corresponding author.

Email address: xshi@berkeley.edu (Xian Shi)
}

leads to in-cylinder fuel stratification is also an effective approach to control combustion phasing and expand engine operation load limits [6]. Despite the fact that the fuel stratification concept has been successfully implemented in various industrial applications, fundamental understanding of stratified flames is still incomplete. When the stratified mixture is ignited, a consequent flame will be established and propagate through the stratified mixture. How fast the stratified flame propagates compared to the corresponding homogeneous flames is a key question to answer as to the determination of combustion duration and emissions. However, the difference between laminar flame speeds of stratified and homogeneous flames is still neither well understood nor quantified.

Researchers have experimentally observed that stratified flames propagate at different speeds compared to homogeneous flames. Karim and Tsang [7] set up a circular pipe where a flame can be initiated from a bottom open end and propagate towards top closed end. A plate valve was used to separate the pipe into two chambers with two different mixtures. A stratified mixture was formed by removing the plate valve. They found that for methane/air mixtures, when the flame propagated from rich mixtures into near stoichiometric mixtures, the observed flame velocities (flame tip moving speed) were generally close to the corresponding quasi-homogeneous flame velocities. However, from stoichiometric to lean, stratified flame velocities were generally 30\% higher. Badr and Karim [8] exploited a similar setup and extended the above conclusion: 
For stratified flames with decreasing velocities, from near stoichiometric towards either lean or rich, flame velocities were higher than homogeneous flames. For stratified flames with increasing velocities, from rich or lean towards near stoichiometric, the measured velocities were close to those of homogeneous flames. However, in their setup, the level of stratification cannot be preciously controlled thus the conclusions were mainly qualitative. Kang and Kyritsis [9, 10] studied a similar stratified flame of methane/air mixtures in a novel burner that allowed accurate control of mixture stratification using convective-diffusive balance of two fresh methane/air mixture streams. They found that from stoichiometric to either leaner or richer, the stratified flame velocities are higher than the homogeneous flames. Furthermore, the increase in flame speed was more prominent when the flame approached the flammability limits and encountered higher equivalence ratio gradients. In a recent experimental study by Balusamy et al. [11], a propane/air flame was ignited in a rich mixture and propagated into a lean mixture in a constant volume chamber. The flame propagation in the lean mixture was found back-supported by ignition in richer conditions, as the flame benefited from the rich composition of the burnt gas compared to that of lean homogeneous flames.

Despite the fruitful observations from experimental studies, there still remain critical questions which cannot be fully resolved by experiments:

1. In a stratified flame, both thermal and mass diffusion processes are different from those in the corresponding homogeneous flames. Experimental evaluation of each process cannot be conducted exclusively thus their comparative impacts on laminar flame speeds of stratified flames were yet understood.

2. Specific experimental setups, including propagation directions and boundary conditions, might introduce additional effects or biases. For example, for flames propagating vertically upwards, the hot burned gases are more buoyant and tend to propagate faster relative to the flame and thereby assist the convective mass transfer of active species ahead of the flame.

3. Flame stretch and curvature undermine the accuracy of measured flame speeds. For example, in [11], due to the initial mixture preparation and ignition setup, an oval contour of flame front was developed instead of an ideal sphere. Consequently, tracking flame front propagation and extrapolation of the unstretched laminar flame speed are problematic.

4. Discrepancy exists between stoichiometry of unburnt mixture and mixtures at flame front, due to preferential diffusion of lighter species. As some lighter but important chemical species and radicals, e.g., $\mathrm{H}, \mathrm{H}_{2}$, diffuse much faster than other species, mixture composition at the flame front can be quite different from that of the corresponding unburnt mixture. Therefore, the structure of a pre-defined stratified mixture based on unburnt mixtures might change during the course of stratified flame propagation.

In order to compensate the above experimental limitations, numerical studies with idealized setups are conducted to reduce the problem complexity and help identify dominant processes which are responsible for the differences between stratified and homogeneous flames.

There have already been several numerical studies investigating the behavior of stratified flames: Cruz et al. [12] performed 1-D unsteady simulations of laminar stratified methane/air flames. They concluded that the propagation of lean-to-rich stratified flames is influenced mainly by heat from burned gases, i.e., thermal effect. For rich-to-lean stratified flames, the propagation is dominated by production and consumption of molecular hydrogen in the rich burnt gas, i.e., chemical effect. However, although the differences between temperature and species distributions at the flame front of stratified flames and of homogeneous flames were reported, an analysis with adequate details about how these diffusion processes compete was not given. Zhou and Hochgreb [13] conducted numerical simulations of methane/air counterflow stagnation flames and confirmed that methane/air stratified flames are primarily dominated by the diffusion of heat under lean conditions, and diffusion of $\mathrm{H}_{2}$ under rich conditions. In contrast, Zhang and Abraham [14] investigated planar stratified methane/air flames. They confirmed that additional diffusion of heat and active species had made rich-to-lean stratified flames faster than corresponding homogeneous flames. More importantly, they studied comparative effect of thermal and species diffusion by considering equal-diffusivity transport model and concluded that species diffusion is more important in increasing flame speeds of stratified mixtures. In a recent numerical study by the authors [15], stratified hydrogen/air laminar flames were investigated. Local flame speeds of rich-to-lean stratified flames were found overall faster than homogeneous flames, which agrees with previous observation. However, through a diffusion speed study, thermal effect was found to be unimportant as thermal diffusion speed is not fast enough to catch up the flame front propagation. The chemical effect was identified through enhanced levels of $\mathrm{H} \mathrm{rad}-$ icals produced in the burnt gas, which diffuse faster enough into the flame front and enhanced the fuel consumption and local heat release rate.

As a continuous endeavor of the previous study [15], this numerical study compares stratified flames of three different hydrocarbon fuels, i.e., methane, propane and $n$-heptane, in a quantitative manner. Specifically, the study focuses on rich-tolean stratified flames and aims to answer:

1. How different are flame properties, such as temperature and species distributions as well as chemical reaction pathways, between stratified and homogeneous flames of different hydrocarbon fuels?

2. Does the trend, that rich-to-lean stratified flames are faster than homogeneous flames, still hold for larger hydrocarbon fuels? If not, what is the corresponding dominant process responsible for the opposite trend?

Numerical simulations of methane, propane and $n$ heptane/air stratified flames are performed and compared to their corresponding homogeneous flames. For one to one comparison, consistency in characterizing flame properties between 
stratified and homogeneous flames is crucial, e.g., location of flame front, reference equivalence ratio and flame speed. Therefore, in this study, all the investigated and relevant flame characteristics are consistently defined and illustrated in later sections. Since stratified flames propagate through varying mixture compositions, the Lewis number of deficient reactant may affect flame stability. As all the simulations are one-dimensional and conduced in a planar geometry, the potential flame instability introduced by curvature is not captured.

\section{Numerical Model and Setup}

A parallel version of the Adaptive Simulation of Unsteady Reacting Flow (ASURF-Parallel) [15, 16], is used to perform transient simulations of stratified and homogeneous flames. The program solves conservation equations of onedimensional, compressible, multi-component reactive flow using finite volume method. Chemical species and elementary chemical reactions are handled by CHEMKIN packages $[17,18]$. A multi-level dynamically adaptive mesh refinement (AMR) algorithm is applied to resolve the reaction front. In addition to the original AMR with thresholds of temperature gradients, refinement based on local equivalence ratio gradients is also implemented in order to resolve the stratification layer. A domain decomposition approach based on Message Passing Interface (MPI) is implemented to speed up the calculation. In this study, a fractional-step procedure is implemented to separate stiff chemistry reaction term from convection and diffusion terms. For the chemistry step, a stiff VODE solver is used. For the transport step, a second-order Runge-Kutta method is utilized for time integration while diffusion and convection are solved through central difference method and HLLC Riemann solver respectively.

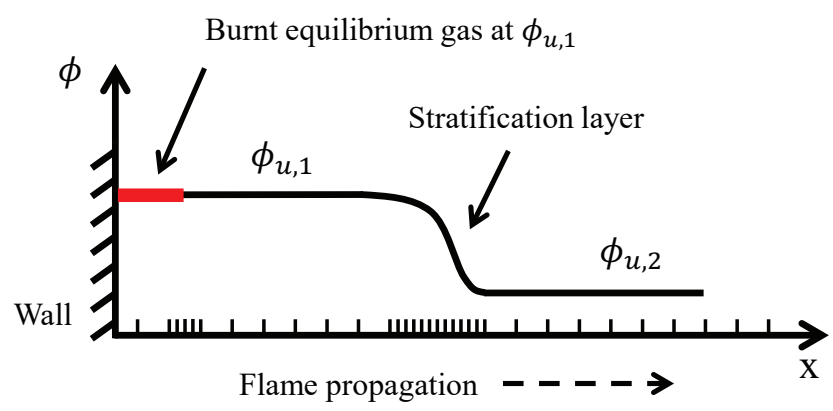

Figure 1: Schematic of stratified flame setup in 1-D planar coordinate.

Figure 1 sketches the numerical setup of a representative stratified flame. The left end is a closed end as zero Dirichlet boundary conditions is applied, while the right end is an open end approximated by zero-gradient Neumann boundary condition. The flame is initialized at the closed end with homogeneous unburnt mixture of equivalence ratio $\phi_{u, 1}$. Ignition is achieved by specifying the burnt equilibrium temperature, velocity and composition profiles of unburnt mixture $\phi_{u, 1}$ at the closed end. Therefore, a consequent propagating flame can be established with least unsteadiness disturbance. The flame propagates through a stratification layer before reaching another homogeneous unburnt mixture of equivalence ratio $\phi_{u, 2}$. The stratification is introduced by specifying a step change in the initial equivalence ratio profile from $\phi_{u, 1}$ to $\phi_{u, 2}$. At the time when the flame front reaches the stratification layer, a smooth structure of stratification, as shown in Fig. 1 can be achieved due to natural mass diffusion process. Laminar flame speeds, in terms of either fuel consumption speeds or flame front propagation speeds, are tracked throughout the stratified flame propagation as a function of reference equivalence ratio at flame front. The transient stratified flame speed results are later compared to the corresponding homogeneous flames. The homogeneous flame results were generated by running entirely homogeneous mixtures with the same setup until the flame speeds no longer change. All the cases were conducted in an 1-D domain of 20 $\mathrm{cm}$ under initial temperature and pressure of $300 \mathrm{~K}$ and $1 \mathrm{~atm}$. A 9-level AMR scheme is applied with $\Delta x_{\max }=0.2 \mathrm{~cm}$ and $\Delta x_{\min }=4 \mu \mathrm{m}$. As a results, the flame front where major heat release occurs is revolved by approximately 250 grids. The time step is $1 \mathrm{~ns}$. Only planar simulations are conducted and hence all the results reported are stretch free. The thickness of stratification layer, which will be defined in the next section, is of the order of $0.1 \mathrm{~cm}$, approximately the same order of flame thickness.

For methane/air flames, a 16-species reduced chemical kinetic mechanism was developed from a short version of GRI3.0 [19] without $\mathrm{NO}_{\mathrm{X}}$ chemistry. For propane/air flames, a 24species reduced mechanism was developed from a 70-species detailed mechanism [20]. For $n$-heptane/air flames, a 32species reduced mechanism was developed from a 65-species skeletal mechanism [21]. All the reduced mechanisms were developed using in-house Computer Assisted Reduction Mechanism (CARM) software [22], including directed relation graph (DRG) method and target search algorithm (TSA). The computed flame speeds of homogeneous flames agree well with experimental data in the literature and the comparisons are included in the supplemental material. A further validation of $n$-heptane reduced chemistry against detailed chemistry is provided as well.

\section{Flame Characteristics}

For a meaningful comparison between stratified flames (SF) and homogeneous flames (HF), SF and HF are compared with the same reference equivalence ratio using the same definition of laminar flame speed. Equivalence ratio at flame front $\phi_{f}$ and of unburnt mixture $\phi_{u}$ are thereby defined to quantify the mixture composition of both SF and HF. The mostly referred laminar flame speed in the literature is $S_{L}$, which represents the unstretched, adiabatic, flame propagation speed relative to unburnt mixtures. However, this ideal definition of laminar flame speed cannot be accurately determined from either experiments nor transient flame simulations. Therefore, alternative flame speed definitions are proposed based on different flame characteristics. In this study, fuel consumption speed, $S_{c}$, and flame 
front propagation speed, $S_{b}$ are defined and used to describe the flame behaviors of SF and HF. The definition of stratification thickness, as to quantify the degree of stratification, is also given.

\subsection{Equivalence ratio at flame front, $\phi_{f}$, and of unburnt mix- ture, $\phi_{u}$}

While the flame front location $x_{f}$ is defined as the location of peak heat release rate, the reference equivalence ratio is defined at flame front on the basis of local element composition instead of unburnt fuel/air ratio [13]:

$\phi_{f}=\frac{X_{H, f}+4 X_{C, f}}{2 X_{O, f}}$

where $X_{H}, X_{C}$ and $X_{O}$ denote the element mole fraction of $\mathrm{H}, \mathrm{C}$ and $\mathrm{O}$ respectively and subscript $f$ denote the location of flame front. The equivalence ratio of unburnt mixture, $\phi_{u}$, can then be calculated using the same definition but considering the composition of unburnt mixture instead of that at flame front. This definition of equivalence ratio is applicable to all hydrocarbon fuels. For homogeneous flames, $\phi_{u}$ can be different from $\phi_{f}$ due to differential diffusion of chemical species. For example, $\phi_{u}$ is equal to 1 for stoichiometric methane/air mixtures. However, $\phi_{f}$ of the same mixture is actually 0.97 due to preferential diffusion of intermediate species such as $\mathrm{H}$ and $\mathrm{H}_{2}$. When comparing SF and HF, both flames share the same $\phi_{f}$ as SF does not have the corresponding unburnt mixture to define $\phi_{u}$.

\subsection{Fuel consumption speed, $S_{c}$}

Laminar flame speeds can be determined through the profile of flame properties, such as temperature variation and species distribution [23], which are accessible in numerical simulations. Fuel consumption speed, $S_{c}$, defines the flame propagation from the perspective of fuel consumption. $S_{c}$ is relatively well-defined and frequently used in turbulent combustion simulations. It can be derived from the mass balance of a stationary flame and written as [24]:

$S_{c}=-\frac{1}{\rho_{u} Y_{F}^{u}} \int_{-\infty}^{+\infty} \dot{\omega}_{F} d x$,

where $Y_{F}^{u}$ is the mass fraction of fuel species in the unburnt gas; $\rho_{u}$ is the unburnt gas density and $\dot{\omega}_{F}$ is the fuel consumption rate. Due to uncertainty in determining $Y_{F}^{b}$ in propagating flames, $Y_{F}^{u}$ is used instead of $Y_{F}^{u}-Y_{F}^{b}$, which was stated in the original theoretical derivation of fuel consumption speed.

\subsection{Flame front propagation speed, $S_{b}$}

Alternatively, laminar flames can be directly characterized by the global behavior of the flame, such as flame front movement speed or equivalent fluid velocity of incoming unburnt mixtures. In this study, flame front propagation speed, $S_{b}$, is defined as the physical moving speed of the flame front:

$S_{b}=\frac{d x_{f}}{d t}$
Subscript $b$ indicates that the flame speed is calculated with reference to burnt gas. Specifically, since the flame is initialized from a closed end and propagates towards an open end, $S_{b}$ is a combination of laminar flame speed relative to unburnt gas and the expansion of burnt gas. Therefore, $S_{b}$ can be related to $S_{L}$ in homogeneous flames as

$S_{b}=\frac{\rho_{u}}{\rho_{b}} S_{L}$,

where $\rho_{u}$ and $\rho_{b}$ represent density of unburnt and burnt mixture. Similar to $Y_{F}^{u}, \rho_{b}$ is not well-defined in stratified flames as well as propagating homogeneous flames. Thus $S_{L}$ cannot be unambiguously derived from the above relation.

\subsection{Stratification thickness, $\delta_{s}$}

As the step change in equivalence ratio is placed at a certain distance away from the closed end, the stratification layer will grow due to mass diffusion before the flame front arrives. In order to quantify the degree of stratification when the flame front reaches the stratified mixture, stratification thickness $\delta_{s}$ is defined and given as following:

$\delta_{S}=\frac{\left|\phi_{u, 1}-\phi_{u, 2}\right|}{\left|\frac{d \phi_{f}}{d x}\right|_{\max }}$.

similar to the flame thickness definition based on peak temperature gradient [25]. Different stratification thicknesses can be achieved by placing the initial step change at different locations relative to the closed end. In this study, the stratification thickness is controlled around $0.1 \mathrm{~cm}$, at the same order of typical hydrocarbon flame thickness. Note that for all three fuels, larger the stratification thickness is, less prominent effect is introduced, while the overall trend holds the same. This observation agrees with the previous findings in [15]. A comparison among stratified flames with different stratification thicknesses and homogeneous flames of methane/air mixtures is provided in the supplemental material.

\section{Results and Discussion}

In this section, rich-to-lean stratified flames, propagating from unburnt mixture of $\phi_{u}=1.60$ to 0.60 , are compared to their corresponding homogeneous flames, at the same equivalence ratio at flame front $\phi_{f}$. Both fuel consumption speeds and flame front propagation speeds are compared. Note that stratified flame is essentially unsteady, as the flame front passes through mixtures with varying compositions. Therefore, the equivalence ratio of upstream burnt gas is no longer the same as that at flame front. The arbitrary burnt gas composition will result in enhanced/reduced diffusion processes between burnt gas and flame front compared to homogeneous flames, especially of intermediate lighter species such as molecular hydrogen. Therefore, the local flame behaviors and laminar flame speeds of stratified flames are different from those of homogeneous flames. Detailed analysis of temperature, species and reaction heat release around the flame front are conducted to explain the trends and identify contributions from diffusion processes and chemical reaction pathways. 


\subsection{Comparison among fuel consumption speeds}

Figure 2 presents the fuel consumption speeds of SF and HF for all three hydrocarbon fuels. Fuel consumption speeds $S_{c}$ are plotted against equivalence ratio at flame front $\phi_{f}$. Transient stratified flames are represented by the solid lines, while the reference homogeneous flames are shown in terms of symbols. The stratified flame first started with the same $S_{c}$ as that of the homogeneous mixture at rich end $\phi_{f} \approx 1.6$, then deviated from homogeneous flames throughout its propagation inside the stratification layer, and gradually converged to the lean end $\phi_{f} \approx 0.6$. For methane/air, the overall trend is that $S_{c}$ of the rich-to-lean SF are enhanced compared to those of HF across the entire stratification layer, which agreed with some previous studies [12]. However, for propane and $n$-heptane, while the enhancement on $S_{c}$ of SF is found on the lean side, an opposite trend is observed on the rich side. The percentage differences between $S_{c}$ of SF and HF with regard to $\mathrm{HF}$ are calculated and presented in Fig.3. From $\phi_{f} \approx 1.6$ to 1.3 , negative percentages for propane and $n$-heptane are observed, indicating decreases in $S_{c}$ of SF. In order to understand this unexpected behavior of propane and $n$-heptane SFs, detailed flame properties are analyzed $\phi_{f} \approx 1.40$, where the maximum discrepancy occurs.

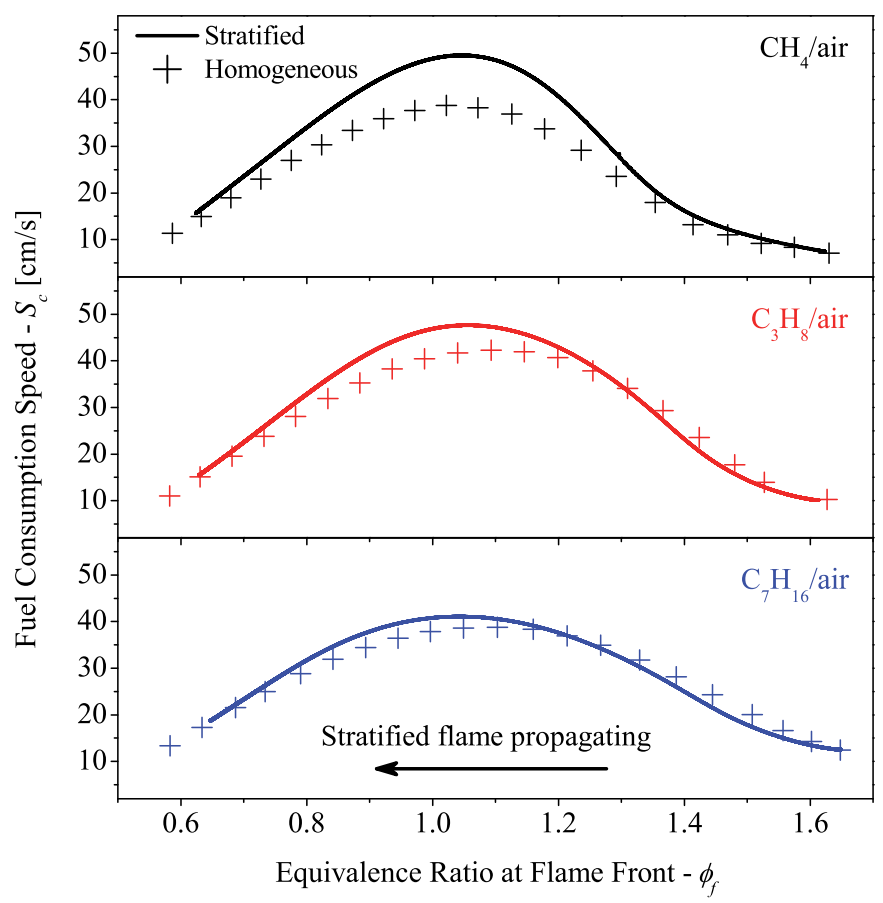

Figure 2: Fuel consumption speeds of the rich-to-lean stratified flame and the corresponding homogeneous flames.

For reference, methane/air mixture is first analyzed. For the homogeneous mixture of $\phi_{u}=1.40, \phi_{f}$ is equal to 1.41 . Figure 4 shows the profiles of computed fuel consumption rates and heat release rates, where $x=0$ is the location of the flame front. Both peak heat release rate and fuel consumption rate of SF are larger than those of HF. The location of peak fuel consumption is also located very close to the flame front. Figures 5 and 6 compare the computed temperature, reactant, product as well as intermediate species profiles of both SF and HF. In Figure

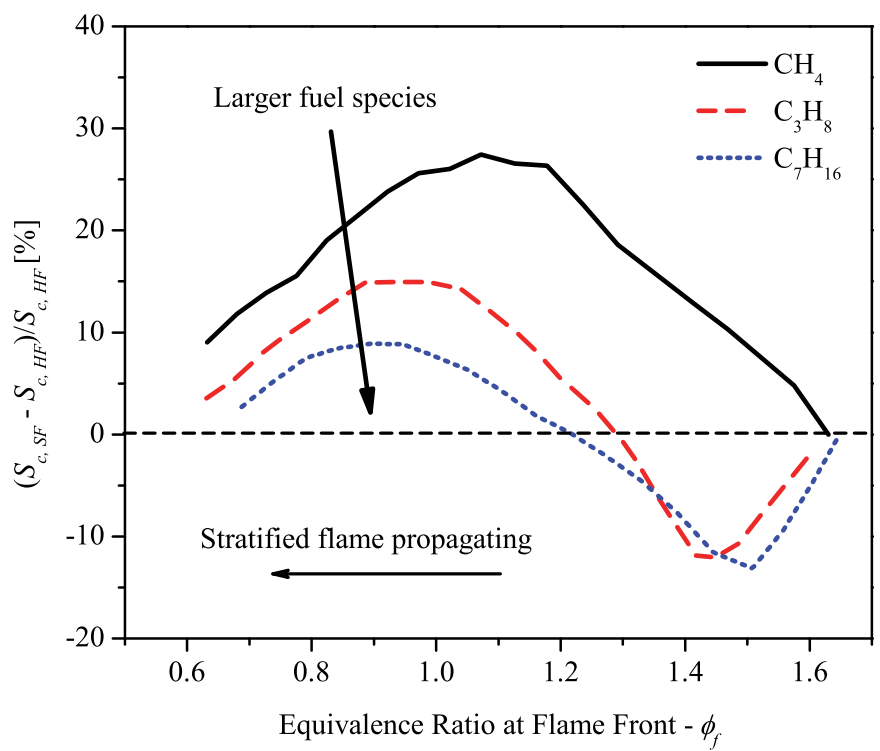

Figure 3: Percentage differences between fuel consumption speeds of the richto-lean stratified flame and the corresponding homogeneous flames.

5 , the temperature at flame front is $\sim 1800 \mathrm{~K}$. On the unburnt side $(x>0)$, the decreasing profile of $\mathrm{CH}_{4}$ seen in the $\mathrm{SF}$ is indicative of that the flame propagates towards a leaner mixture. A higher level of $\mathrm{H}_{2} \mathrm{O}$ is found in the unburnt mixture of SF, which exhibits the same trend as temperature: the unbunrt temperature of SF near the flame front is notably higher (about 150 $K$ ) than that of HF. Similarly in Figure 6, higher levels of $\mathrm{H}_{2}$ and $\mathrm{CO}$ are also found in unburnt mixture of SF. As there is no discernible heat release in the preheat zone of SF $(x>0.025 \mathrm{~cm})$ thus no local formation of products, these species $\left(\mathrm{H}_{2} \mathrm{O}, \mathrm{H}_{2}\right.$, $\mathrm{CO})$ must have diffused from the flame front or burnt gas into the unburnt mixture. A higher temperature of the unburnt mixture is achieved due to high enthalpy carried by these species. In Figure 6, more $\mathrm{H}$ and $\mathrm{OH}$ radicals are found near the flame front in SF than HF, which agree with the observation of higher heat release rate and fuel consumption rate in SF. This enhancement in radical pool resulted from preferential diffusion of molecular hydrogen and was referred to as the chemical effect of SF from previous studies [12]. Note that the burnt gas temperature of $\mathrm{SF}$ is lower than that of HF in Fig.5, which should have led to a decreased unburnt gas temperature of SF due to reduced thermal diffusion. However, the unburnt gas temperature of SF is higher due to enhanced radical and product diffusion therefore the impact of chemical effect is dominant compared to thermal effect [15]. Although not presented in this paper, the results around $\phi_{f}=1$ where the enhancement is most prominent show that substantially more hydrogen (almost twice as much), water as well as consequent higher unburnt gas temperature were observed in the stratified flame. This observation confirmed the dominance of chemical effect. In all, fuel consumption speeds of SF are higher than those of HF for methane/air mixtures, due to enhanced radical pool at the flame front and increased unburnt mixture temperature.

Another evidence of the chemical effect is revealed by the 


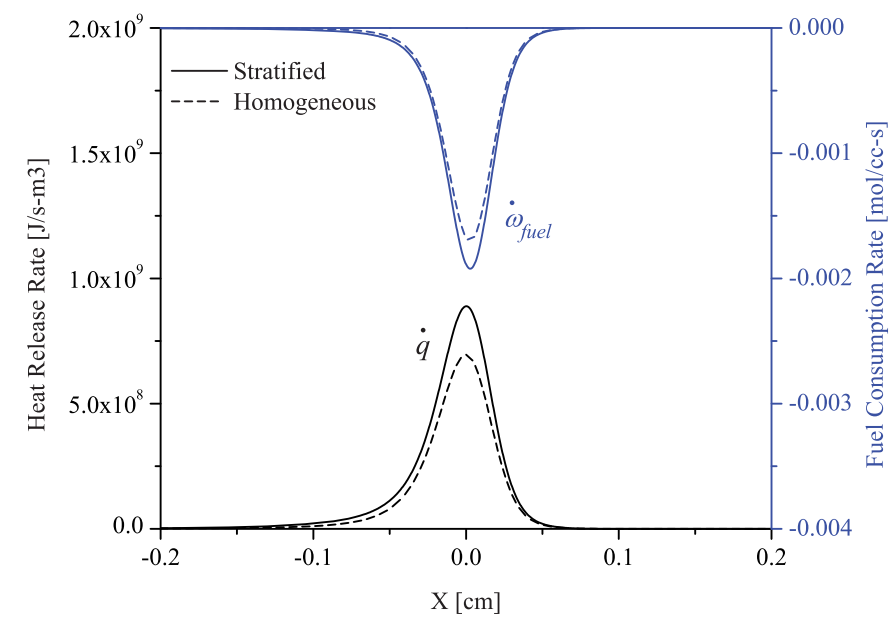

Figure 4: Heat release rates and fuel consumption rates in methane/air stratified and homogeneous flames, at $\phi_{f}=1.41$.

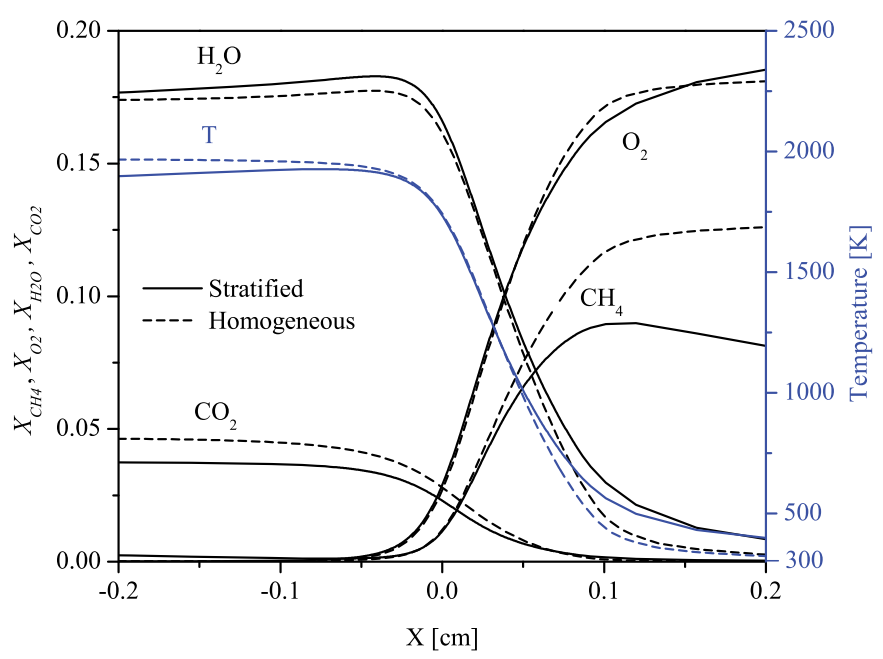

Figure 5: Temperature, mole fractions of reactants and products in methane/air stratified and homogeneous flames, at $\phi_{f}=1.41$.

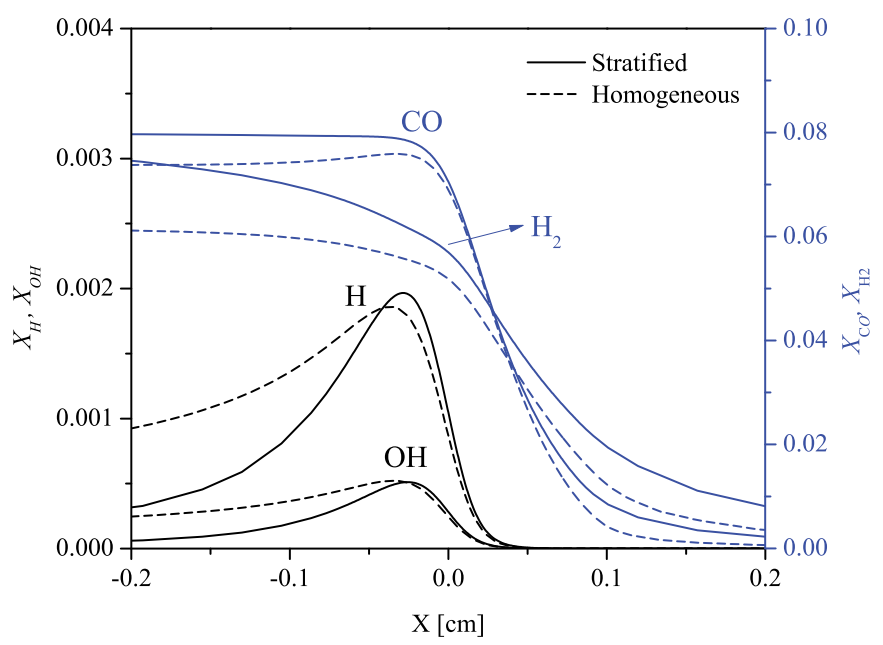

Figure 6: Mole fractions of intermediate species and radicals in methane/air stratified and homogeneous flames, at $\phi_{f}=1.41$. reaction heat release analysis. Figure 7 shows the differences between heat release rates of each reaction in methane/air SF and HF, plotted against reaction index number. The reaction heat release rate is calculated as the integration of heat release of one particular reaction over the entire calculation domain divided by the domain length. A positive value means that this reaction releases/absorbs more heat in SF than that in $\mathrm{HF}$. Reactions A-G substantially releases/absorbs more heat in SF than that in HF and indicated that the overall heat release behavior in SF is more active compared to HF. All major reactions are associated with radicals $(\mathrm{H}$ and $\mathrm{OH})$ or reactive intermediate species $\left(\mathrm{H}_{2}, \mathrm{CO}\right.$ and $\left.\mathrm{CH}_{3}\right)$ that are formed more in SF.

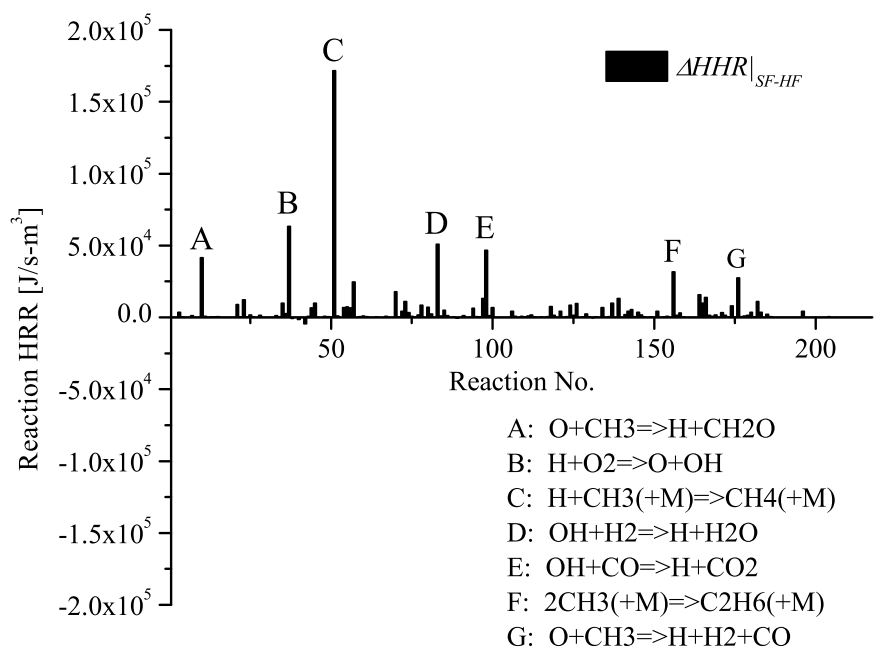

Figure 7: Difference between absolute heat release rates of reaction steps in methane/air stratified and homogeneous flames, at $\phi_{f}=1.41$.

A similar analysis is conducted for propane/air HF and SF. Detailed flame properties are analyzed at $\phi_{f}=1.42$ (corresponding to HF with the unburnt mixture of $\phi_{u}=1.40$ ). As propane/air flames at $\phi_{f}=1.42$ have higher flame speeds $(\sim 23 \mathrm{~cm} / \mathrm{s})$ compared to methane/air flames $(\sim 13 \mathrm{~cm} / \mathrm{s})$, both profiles of heat release rates and fuel consumption rates of propane/air flames shown in Fig.8 are thinner compared to methane/air flames. In Fig.8, while the peak fuel consumption rate of SF is clearly smaller than that of $\mathrm{HF}$, heat release rates of both flames are almost identical. Although the peak heat release rate of SF is smaller than HF, the total heat release rate of SF is still slightly higher than HF since SF releases more heat on the burnt side of the peak location. The peak locations of heat release rate and fuel consumption rate no longer coincide, showing that major fuel consumption occurs on the unburnt side of major heat release. As fuel decomposition reaction steps in propane/air flames have lower activation temperatures compared to methane/air flames, fuel consumption peaks at a lower temperature, $\sim 1400 \mathrm{~K}$.

Temperature and major species profiles in Fig.9 show similar trends as those of methane/air flames. Unburnt mixture of SF has higher temperatures than HF. As a result, fuel consumption of SF occurs slightly earlier than HF in Fig.8, while mole fraction of $\mathrm{C}_{3} \mathrm{H}_{8}$ decreases earlier as well in Figure 9. For radical 
distributions in Fig.10, while mole fractions of $\mathrm{CO}$ and $\mathrm{H}_{2}$ are still higher in SF similar to methane, mole fractions of $\mathrm{H}$ and $\mathrm{OH}$ near the flame front of propane SF are lower than those of HF, opposite to methane. Therefore, a reduced level fuel consumption rate and $S_{c}$ is expected for SF due a reduced pool of key radicals.

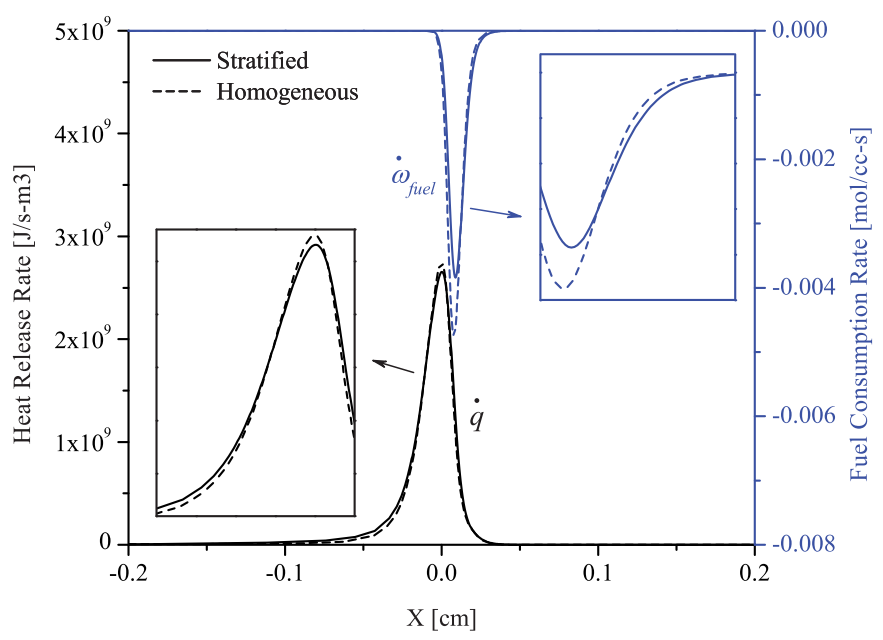

Figure 8: Heat release rates and fuel consumption rates in propane/air stratified and homogeneous flames, at $\phi_{f}=1.42$.

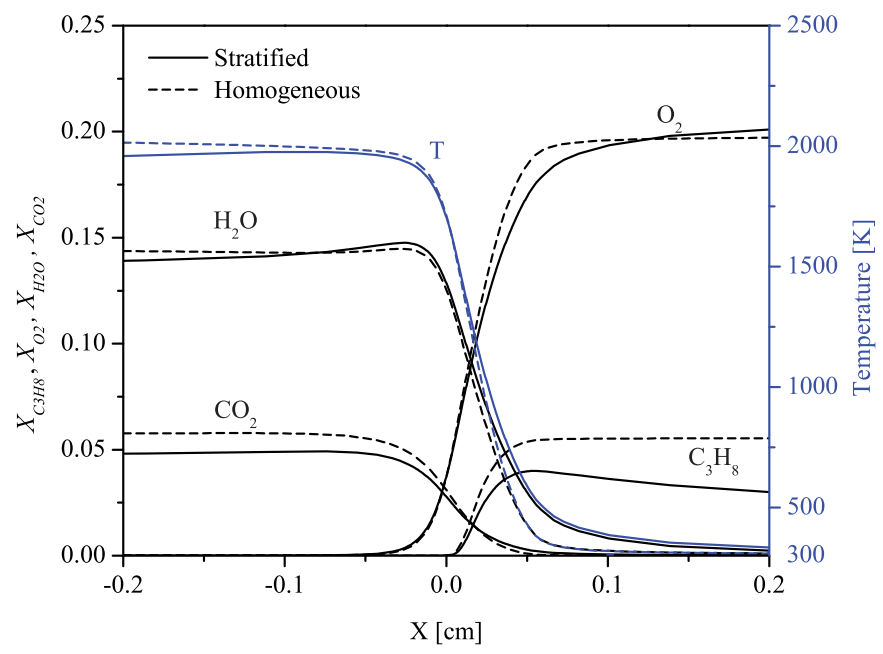

Figure 9: Temperature, mole fractions of reactants and products in propane/air stratified and homogeneous flames, at $\phi_{f}=1.42$.

In the reaction heat release analysis shown in Fig.11, Reaction $\mathrm{A}$, recombination of $\mathrm{H}$ and $\mathrm{CH}_{3}$, and Reaction $\mathrm{B}$, wet route of $\mathrm{CO}$ oxidation, release more heat in SF than $\mathrm{HF}$ and they consume more $\mathrm{H}$ and $\mathrm{OH}$ radicals respectively. In contrast, there are fewer reactions of radical production in propane/air SF compared to methane/air SF. Therefore, in propane/air SF, intermediate hydrocarbon species generated from richer mixtures consume radicals in the burnt gas, leading to decreased levels of these key radicals and consequently reduced fuel consumption rate of SF. These intermediate hydrocarbon species are mainly seen in rich flames of larger hydrocarbon fuels. The

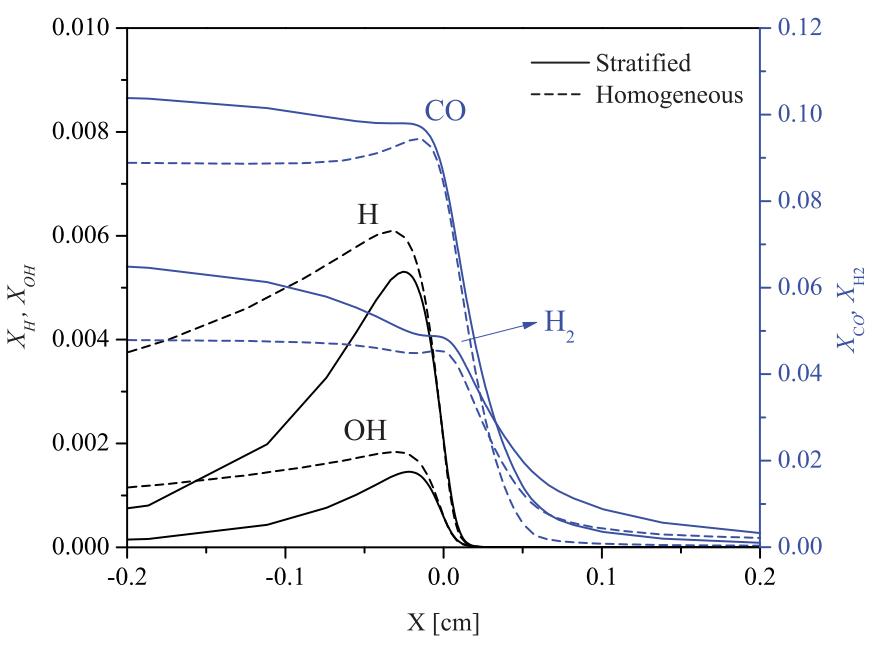

Figure 10: Mole fractions of intermediate species and radicals in propane/air stratified and homogeneous flames, at $\phi_{f}=1.42$.

enhancement in fuel consumption speed seen in methane/air SF is thereby suppressed.

Due to the nature of complicated chemical pathways for larger hydrocarbon fuels, the heat release not only comes from reactions related to fuel decomposition around flame front, but also the reactions between small hydrocarbon molecules and reactive radicals on the burnt side of flame front. In stratified flames of propane/air mixtures, the heat release is enhanced through the latter reactions so that radicals are consumed on the burnt side and not as available for fuel decomposition compared to HF. Although $S_{c}$ is reduced in SF, the total heat release rate of SF is still slightly higher indicated by Fig. 11.

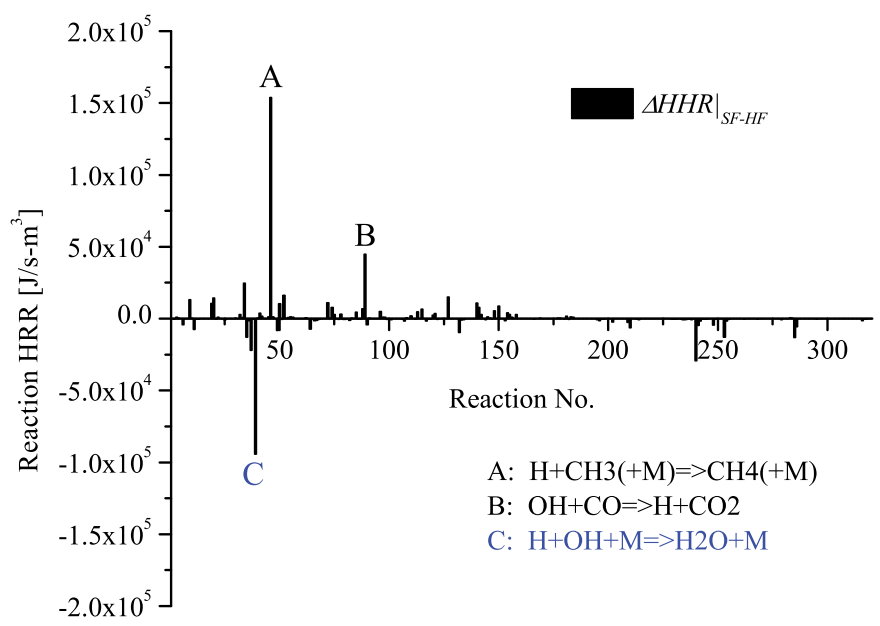

Figure 11: Difference between absolute heat release rates of reaction steps in propane/air stratified and homogeneous flames, at $\phi_{f}=1.42$.

The computed $n$-heptane/air flames exhibit a very similar trend as propane/air flames. Therefore, only the heat release reaction pathway analysis is provided for $n$-heptane/air flames. For unburnt mixture $\phi_{u}=1.40$ of $n$-heptane/air mixtures, $\phi_{f}$ is equal to 1.44. In Figure 12, besides Reaction $\mathrm{A}$ and $\mathrm{C}$ which appear in propane/air flame analysis, Reaction B and D which 
involve in $\mathrm{CH}_{2} \mathrm{O}$ and $\mathrm{CH}_{2} \mathrm{CO}$ consuming $\mathrm{H}$ release more heat are found more active in SF than HF. Small intermediate hydrocarbon species such as $\mathrm{CH}_{2} \mathrm{O}$ and $\mathrm{CH}_{2} \mathrm{CO}$ remain in the burnt gas composition of richer mixtures and react with available radicals. It again indicates that the enhancement in fuel consumption speed is suppressed as radicals are consumed by the intermediate species of richer mixtures. The total heat release rate is still higher in SF, similar to propane.

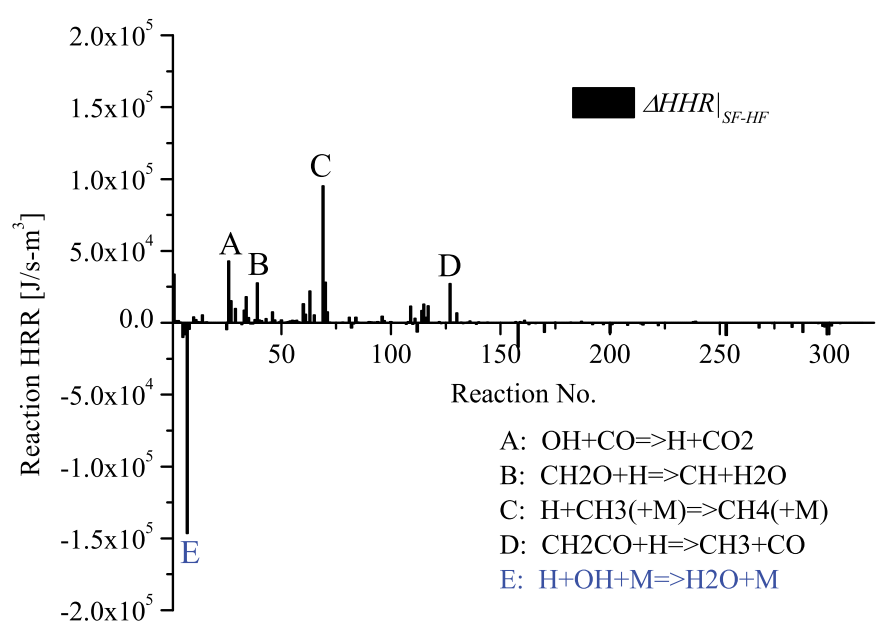

Figure 12: Difference between absolute heat release rates of reaction steps in $n$-heptane/air stratified and homogeneous flames, at $\phi_{f}=1.44$.

\subsection{Comparison among flame front propagation speeds}

Comparisons of the computed flame front propagation speeds $S_{b}$ are presented in Fig.13 and 14. Different from the fuel consumption speed, the flame front propagation speed represents the local heat release behavior as well as the expansion of burnt gas. Moreover, the flame front propagation speed can be experimentally measured, facilitating comparisons between numerical results and experimental observations. For all three fuels, $S_{b}$ of SF are consistently faster than those of HF throughout the entire stratification layer. The trends in $S_{b}$ are consistent with those in $S_{c}$ between SF and HF of all three fuels on the lean side, due to preferential diffusion of molecular hydrogen, i.e. chemical effect. In contrast, $S_{b}$ of SF on the rich sides are also enhanced compared to $\mathrm{HF}$, different from the $S_{c}$ results of propane and $n$-heptane. Recall that although the fuel consumption speeds are reduced, the total heat release rates are still faster in rich SF of propane and $n$-heptane compared to their corresponding HF. According to the previous hydrogen stratified flame study [15], the transient expansion of burnt gas can introduce hydrodynamic effect, which changes the local velocity field thus influencing the flame front propagation speeds. When the flame front accelerates, propagating from rich to stoichiometric mixtures, not only is the unburnt gas further pushed away by the flame front, the burnt gas is also compressed towards the closed end, resulting in slightly negative fluid velocities. The flame front propagation speed tends to decrease due to consequent negative velocity field of burnt gases near the wall and vice versa. For SFs of three fuels in this study, the magnitude of flame acceleration/declaration is much smaller compared to hydrogen/air SF, as the velocity field of burnt gas remain negligibly small during the stratified flame propagation. Therefore, the hydrodynamic effect is negligible compared to the enhanced heat release due to chemical effect.

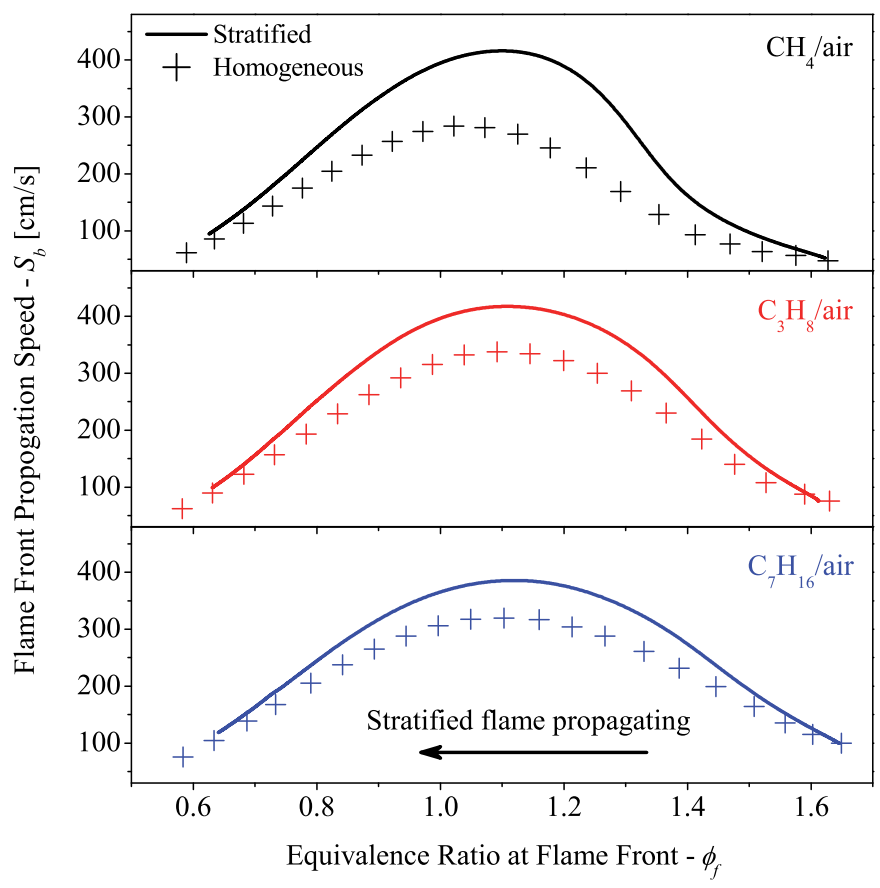

Figure 13: Flame front propagation speeds of the rich-to-lean stratified flame and the corresponding homogeneous flames.

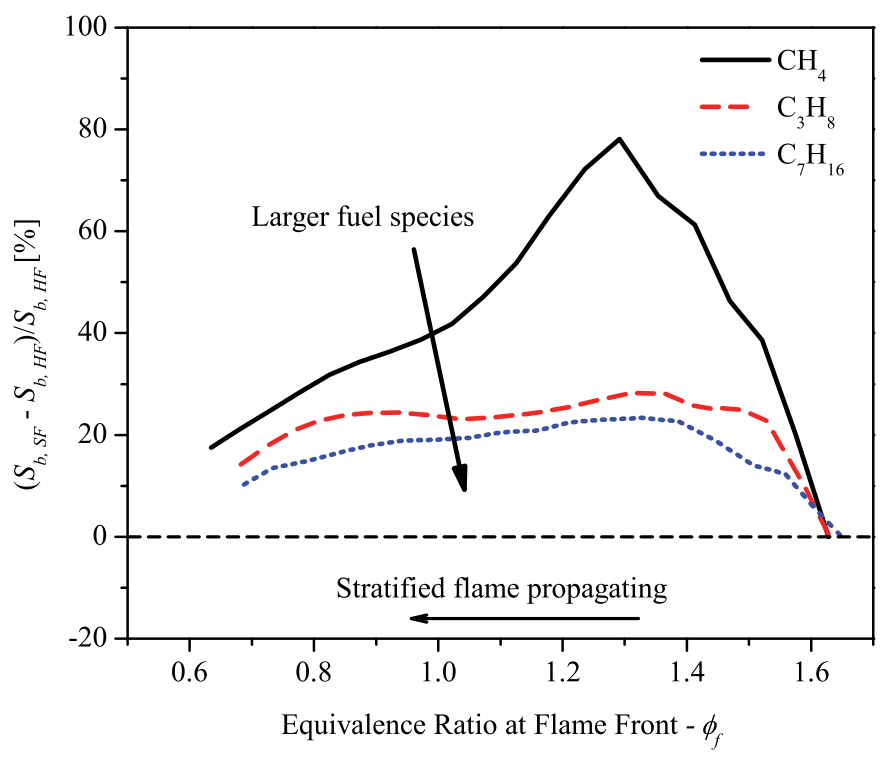

Figure 14: Percentage differences between flame front propagation speeds of the rich-to-lean stratified flame and the corresponding homogeneous flames. 


\subsection{Effect of intermediate molecular hydrogen and its prefer- ential diffusion}

Despite the discrepancy between the results of $S_{c}$ and $S_{b}$ when comparing $\mathrm{SF}$ and $\mathrm{HF}$ on the rich side, a similar trend was shown when comparing different fuel species. In both Fig. 3 and Fig.14, the percentage difference between SF and HF decreases in the sequence of methane, propane and $n$-heptane. Recall that all three stratified flames have about the same stratification thickness. Therefore, the reduced trend on impacts of stratification with larger fuel species should be related to specific fuel properties.

According to the previous analysis, the enhancement in fuel consumption speeds of stratified mixtures results from the chemical effect, where lighter species such as $\mathrm{H}_{2}$ diffuse ahead of the flame front. Figure 15 plots the mole fraction of molecular hydrogen $\left(\mathrm{H}_{2}\right)$ on the unburnt side of flame front (half flame thickness from flame front into the unburnt mixture) against equivalence ratio at flame front. The rich-to-lean stratified flame and the corresponding homogeneous flames are shown in terms of lines and symbols respectively. For homogeneous flames of all three fuels, little amount of hydrogen was produced under lean conditions. On the rich side, methane/air flames produced most amount of hydrogen, followed by propane and $n$-heptane in part due to their lower $\mathrm{H} / \mathrm{C}$ ratio of fuel molecule. As larger fuel tends to produce less intermediate molecular hydrogen under rich conditions, the corresponding stratified flames contain less hydrogen in the burnt gas to assist the local heat release. As a result, the enhancement in fuel consumption speeds of propane and $n$-heptane are not as strong as methane.

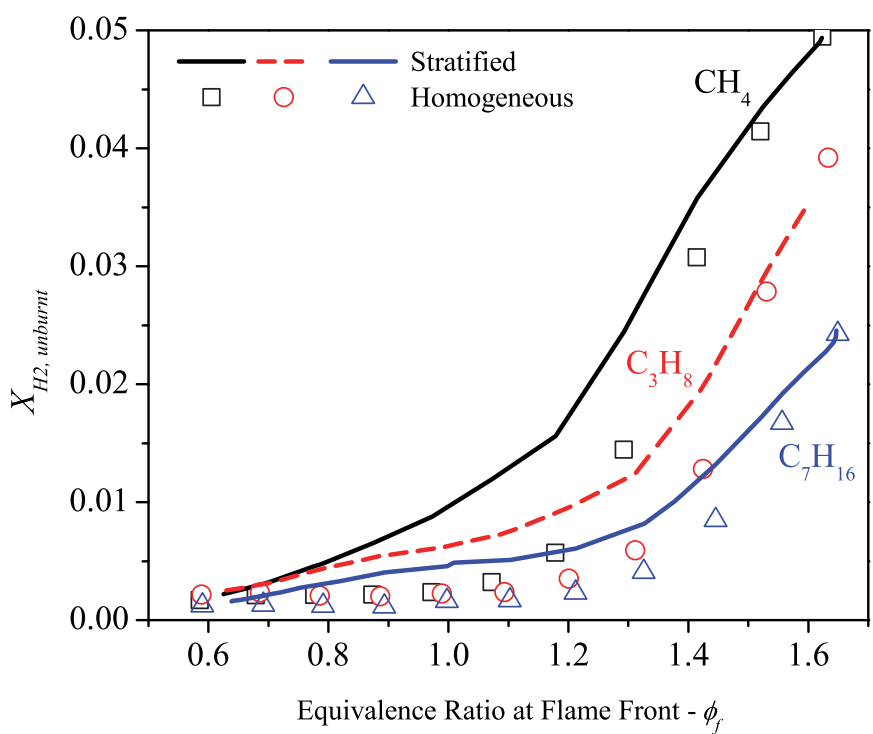

Figure 15: Mole fraction of molecular hydrogen on the unburnt side of flame front, for both the rich-to-lean stratified flame and the corresponding homogeneous flames.

Although the amount of intermediate hydrogen produced in rich mixtures has been found to decrease in mixtures of larger fuel species, it does not necessarily indicate that the enhancement in fuel consumption speeds scales with hydrogen since combustion chemistry is different. Figure 16 quantifies the enhancement of homogeneous laminar flame speeds due to hydrogen addition for all three fuels. The computed results come from simulations of homogeneous mixtures with different amount of initial hydrogen addition. Lean $\left(\phi_{u}=0.7\right)$, stoichiometric $\left(\phi_{u}=1.0\right)$ and rich $\left(\phi_{u}=1.3\right)$ conditions are all tested. For each condition, hydrogen is added into the initial unburnt mixture and the overall equivalence ratio of unburnt mixture based on element composition (Eq.1) is kept constant. The enhancement is evaluated by comparing laminar flame speeds of hydrogen-added and hydrogen-free mixtures. The enhancement percentage is plotted against the mole fraction of hydrogen in the initial unburnt mixture. According to Fig.16, for the same amount of hydrogen addition, the enhancement is found to be stronger in methane compared to propane and $n$-heptane, for all investigated initial mixture compositions.

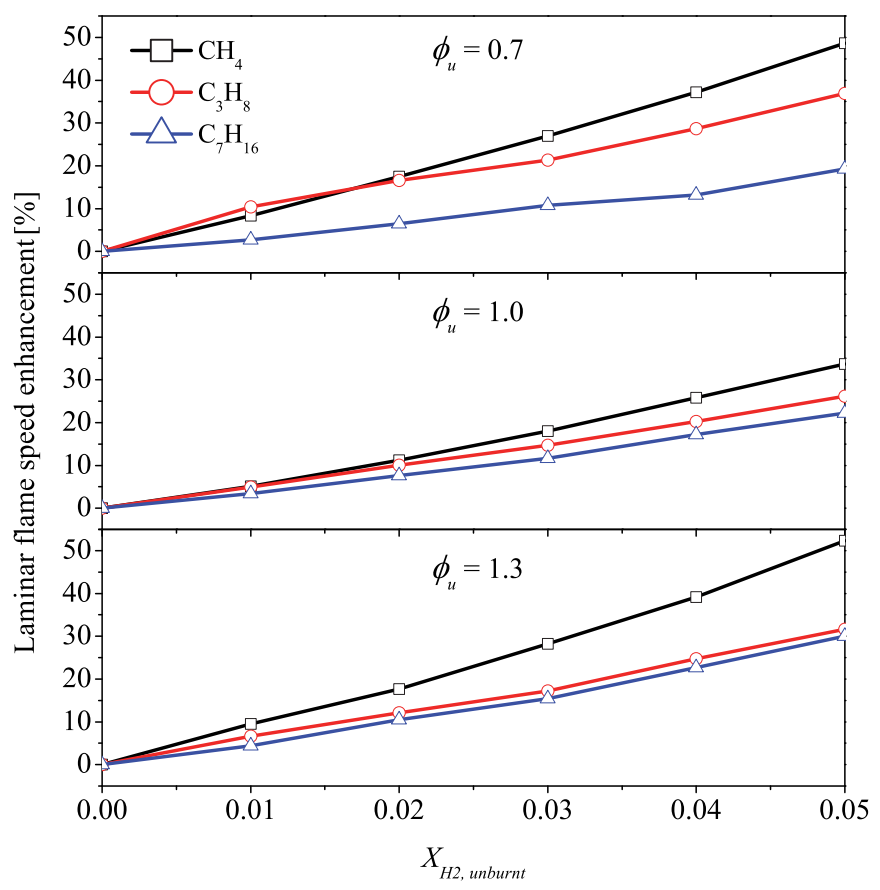

Figure 16: Enhancement of laminar flame speeds with hydrogen addition for methane, propane and $n$-heptane homogeneous flames; $\mathrm{X}_{\mathrm{H} 2 \text {, unburnt }}$ represents mole fraction of hydrogen in initial homogeneous unburnt mixtures.

Therefore, Figure 15 and 16 together indicate that for fuels with a high $\mathrm{H} / \mathrm{C}$ ratio such as methane, more intermediate hydrogen are being generated from its rich mixtures. In a rich-tolean stratified flame, due to preferential diffusion of hydrogen, this enhanced level of hydrogen molecules assisted the local heat release, leading to a even more enhanced laminar flame speed compared to the corresponding homogeneous flames.

\subsection{Identification of mixture properties for stratified flames}

Although equivalence ratio at flame front $\phi_{f}$ is able to evaluate the overall stoichiometry, the ratio between hydrogen and carbon elements can still vary. For rich-to-lean SF, the enhanced level of $\mathrm{H}_{2}$ and $\mathrm{H}_{2} \mathrm{O}$ increased the amount of hydrogen element at flame front. As a result, the mixture composition 
of stratified flame is not exactly the same as that of the corresponding homogeneous flame even with equal $\phi_{f}$. Moreover, the mixture composition of stratified flames with different stratification thicknesses may also vary from each other. Therefore, equivalence ratio at the flame front is inadequate to identify the exact mixture composition of stratified flames.

Figure 17 plots the ratio between hydrogen and carbon elements of SF and HF for all three fuels. The observed trend is consistent compared to the results of flame front propagation speed $S_{b}$ in Fig.14. This result further confirms that the enhanced flame front propagation speeds of rich-to-lean stratified flames result from the chemical effect due to preferential diffusion of molecular hydrogen. For model development purpose, the $\mathrm{H} / \mathrm{C}$ ratio combined with equivalence ratio at flame front may be used together to identify specific stratified flames.

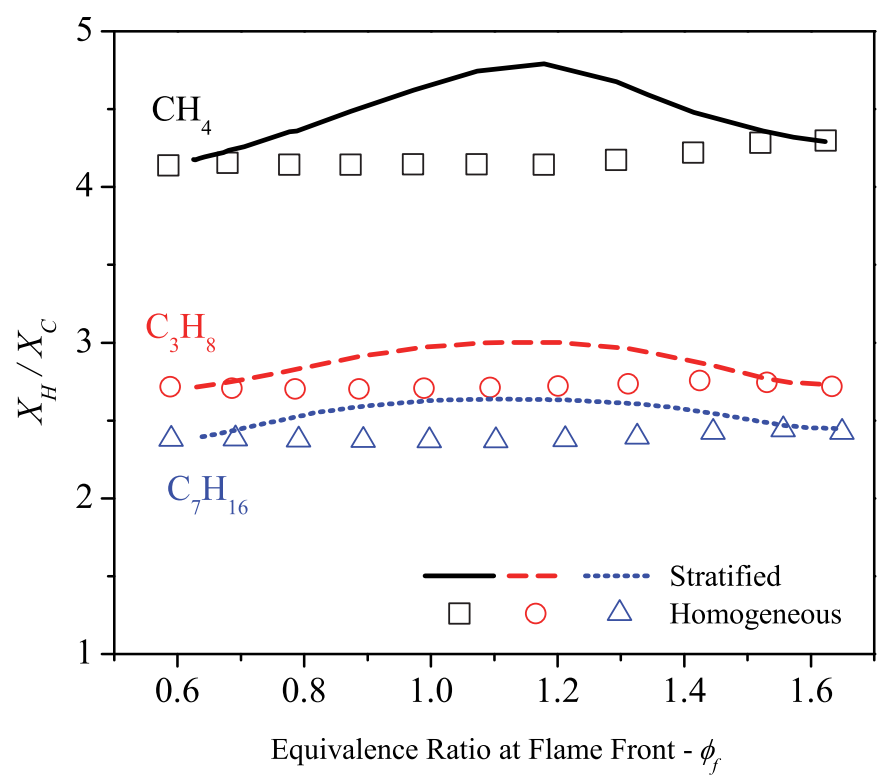

Figure 17: $\mathrm{H} / \mathrm{C}$ ratio at flame front of the rich-to-lean stratified flame and the corresponding homogeneous flames.

\section{Conclusions}

Computed rich-to-lean stratified flames of three different hydrocarbon fuels are compared to their corresponding homogeneous flames in terms of fuel consumption speeds and flame front propagation speeds. The following conclusions are drawn:

1. For fuel consumption speeds, the stratified flame of methane/air is overall faster than homogeneous flames, due to the enhanced chemical activities by more radicals from burnt products of rich mixtures. In comparison, the rich-to-lean stratified flames of both propane/air and $n$ heptane/air flames have slightly lower fuel consumption speeds compared to those of homogeneous flames on the rich side. As reactive radicals such as $\mathrm{H}$ and $\mathrm{OH}$ react with intermediate hydrocarbon species in rich burnt gases, the overall fuel consumption rates of stratified flames are reduced due to lack of radicals at flame front.
2. For flame front propagation speeds, the stratified flames of all three hydrocarbon fuels are found faster than their corresponding homogeneous flames. For methane/air stratified flames, the enhancement of flame front propagation speeds resulted from the chemical effect - preferential diffusion of molecular hydrogen. For propane and $n$-heptane, while the enhancement on the lean side consistently resulted from chemical effect, the enhancement on the rich side resulted from enhanced total heat release rate for those reactions between intermediate species from burnt gas and key radicals $(\mathrm{H}, \mathrm{OH})$ release more heat on the burnt side of flame front. The hydrodynamic effect is found unimportant for stratified flames of these three fuels.

3. For both fuel consumption speeds and flame front propagation speeds, stronger enhancement of stratified laminar flame speeds is observed in methane/air mixtures. More intermediate hydrogen are generated in the rich mixtures of methane, while the corresponding enhancement on laminar flame speeds due to same amount of hydrogen addition is also stronger in methane/air mixtures.

4. Equivalence ratio at flame front is insufficient to define a specific stratified flame as the amount of carbon and hydrogen elements can still vary. The $\mathrm{H} / \mathrm{C}$ ratio along with equivalence ratio at the flame front may be used to provide an improved identification of the exact mixture composition of stratified flames.

\section{Acknowledgments}

This work was supported by the National Science Foundation and U.S. Department of Energy under award CBET-1258653. The authors thank Professor Zheng Chen at Peking University for providing the numerical solver A-SURF.

\section{Appendix A. Supplementary data}

Supplementary data is attached to this manuscript after reference.

\section{References}

[1] R. D. Reitz, Directions in internal combustion engine research, Combust. Flame 160 (2013) 1-8.

[2] A. C. Alkidas, Combustion advancements in gasoline engines, Energy Convers Manage 48 (2007) 2751-2761.

[3] C. Park, S. Kim, H. Kim, Y. Moriyoshi, Stratified lean combustion characteristics of a spray-guided combustion system in a gasoline direct injection engine, Energy 41 (2012) 401-407.

[4] M. Aliramezani, I. Chitsaz, A. A. Mozafari, Thermodynamic modeling of partially stratified charge engine characteristics for hydrogen-methane blends at ultra-lean conditions, International Journal of Hydrogen Energy 38 (2013) 10640-10647.

[5] M. Sjöberg, J. E. Dec, Smoothing HCCI Heat-Release Rates Using Partial Fuel Stratification with Two-Stage Ignition Fuels, SAE Technical Paper 2006-01-0629.

[6] Z. Zheng, M. Yao, Charge stratification to control HCCI: Experiments and CFD modeling with n-heptane as fuel, Fuel 88 (2009) 354-365. 
[7] G. A. Karim, P. Tsang, Flame Propagation Through Atmospheres Involving Concentration Gradients Formed by Mass Transfer Phenomena, J. Fluids Eng. 97 (1975) 615.

[8] O. Badr, G. Karim, Flame Propagation in Stratified Methane-Air Mixtures, J. Fire Sci. 2 (1984) 415-426.

[9] T. Kang, D. C. Kyritsis, Methane Flame Propagation in Compositionally Stratifid Gases, Combust. Sci. Technol. 177 (2005) 2191-2210.

[10] T. Kang, D. C. Kyritsis, Departure from quasi-homogeneity during laminar flame propagation in lean, compositionally stratified methane-air mixtures, Proc. Combust. Inst. 31 (2007) 1075-1083.

[11] S. Balusamy, A. Cessou, B. Lecordier, Laminar propagation of lean premixed flames ignited in stratified mixture, Combust. Flame 161 (2014) 427-437.

[12] A. D. Cruz, A.M.Dean, J.M.Grenda, A numerical study of the laminar flame speed of stratified methane/air flames, Proc. Combust. Inst. 28 (2000) 1925-1932.

[13] R. Zhou, S. Hochgreb, The behaviour of laminar stratified methane/air flames in counterflow, Combust. Flame 160 (2013) 1070-1082.

[14] J. Zhang, J. Abraham, A numerical study of laminar flames propagating in stratified mixtures, Combust. Flame 163 (2016) 461-471.

[15] X. Shi, J.-Y. Chen, Z. Chen, Numerical study of laminar flame speed of fuel-stratified hydrogen/air flames, Combust. Flame 163 (2016) 394-405.

[16] Z. Chen, Studies on the Initiation, Propagation , and Extinction of Premixed Flames, Ph.D. thesis (2009).

[17] R. Kee, G. Dixon-Lewis, J. Warnatz, M. Coltrin, J. Miller, Chemkin: The chemkin transport database, Report No. SAND86-8246, Sandia National Laboratories, Livermore, CA, USA.

[18] R. Kee, F. Rupley, J. Miller, Chemkin: The chemkin thermodynamic database., Report No. SAND87-8215, Sandia National Laboratories, Livermore, CA, USA.

[19] G. P. Smith, D. M. Golden, M. Frenklach, N. W. Moriarty, B. Eiteneer, M. Goldenberg, C. T. Bowman, R. K. Hanson, S. Song, J. William C. Gardiner, V. V. Lissianski, Z. Qin, GRI-Mech 3.0 (1999). URL http: //www.me.berkeley.edu/gri_mech

[20] Z. Qin, V. V. Lissianski, H. Yang, W. C. Gardiner, S. G. Davis, H. Wang, Combustion chemistry of propane: A case study of detailed reaction mechanism optimization, Proc. Combust. Inst. 28 (2000) 1663-1669.

[21] A. Smallbone, W. Liu, C. Law, X. You, H. Wang, Experimental and modeling study of laminar flame speed and non-premixed counterflow ignition of n-heptane, Proc. Combust. Inst. 32 (2009) 1245-1252.

[22] J.-Y. Chen, A General Procedure for Constructing Reduced Reaction Mechanisms with Given Independent Relations, Combust. Sci. Technol. 57 (1988) 89-94.

[23] C. K. Law, Combustion physics, Cambridge University Press, 2006.

[24] T. Poinsot, T. Echekki, M. G. Mungal, A Study of the Laminar Flame Tip and Implications for Premixed Turbulent Combustion, Combust. Sci. Technol. 81 (1992) 45-73.

[25] C. Law, C. Sung, Structure, aerodynamics, and geometry of premixed flamelets, Progress in Energy and Combustion Science 26 (2000) 459 505 . 